\title{
Hallazgo de Batrachyla antartandica Barrio 1967 en isla Riesco
}

\author{
Record of Batrachyla antartandica Barrio 1967 in Riesco Island
}

Gregor J. Stipicic ${ }^{1,2}$, Gabriela Simonetti-Grez ${ }^{2}$,

Sergio Fortes ${ }^{3}$, Claudio Magallanes ${ }^{4}$ \& Javier A. Simonetti ${ }^{2,5}$

Los hallazgos de nuevas localidades $y$ ampliaciones en las distribuciones de anfibios son un fenómeno común en Chile (Correa et al. 2016). Tal es el caso de la rana jaspeada, Batrachyla antartandica Barrio 1967. Descrita con especímenes provenientes de Puerto Blest $\left(41^{\circ} 01^{\prime} \mathrm{S}, \quad 71^{\circ} 48^{\prime} \mathrm{O}\right)$ y Lago Frías $\left(41^{\circ} 03^{\prime} \mathrm{S}\right.$, $\left.71^{\circ} 47^{\prime} \mathrm{O}\right)$, Argentina, y Chamiza (41⒋' $\left.72^{\circ} 85^{\prime} \mathrm{O}\right)$, Puerto Montt, Chile (Barrio, 1967), desde su descripción, en este último país ha sido registrada en el Cerro Mirador, Región de Los Ríos (4008'S, 7355'O) (Formas \& Pugin, 1971); Isla Virtudes, Región de Magallanes y Antártica Chilena

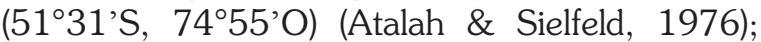
Parque Nacional Vicente Pérez Rosales, Región de Los Lagos (41 $\left.{ }^{\circ} 05^{\prime} S, 72^{\circ} 30^{\prime} \mathrm{O}\right)$ (Penna et al. 1997); Puyehue, Región de Los Lagos (40³5'S, 72²0’O) (Formas, 1979; Penna, 1997); Isla Juan Stuven, Región de Aysén (4755'S, 7452'O) (Díaz-Páez \& Young, 2003); Mehuin (39²6'S $73^{\circ} 12^{\prime}$ O) (Úbeda et al. 2004) y Fundo San Pablo de Tregua, ambos en la Región de Los Ríos (39 35'S, 72 04'O) (Cuevas \& Ugarte, 2008); seno Edimburgo (4905'S, 74²2'O) y Puerto Edén, ambas localidades en la Región de Magallanes y Antártica Chilena (4909'S, 74²7’O) (Asencio et al. 2009), todos registros que amplían su distribución conocida. En esta nota reportamos la presencia de B. antartandica en Cabo León, Parque Nacional Kawésqar, Isla Riesco, Región de Magallanes y Antártica Chilena (5240'S, $\left.72^{\circ} 10^{\prime} \mathrm{O}\right)$, lo que representa una extensión de aproximadamente $121 \mathrm{~km}$ en su límite meridional.

En el marco de la filmación del documental
"Parque Nacional Kawésqar. Refugio de vida", realizado por la Asociación Kauyeken durante enero de 2018, se detectó un ejemplar juvenil de B. antartandica. El espécimen aparece al 1'30" de dicho documental ${ }^{1}$. Al no disponerse de permisos de colecta, el ejemplar solamente fue fotografiado (Fig. 1). El individuo medía aproximadamente 26 $\mathrm{mm}$, de cuerpo esbelto con extremidades delgadas, de dedos libres, largos y finalizando en forma de espátula, cabeza deprimida, de hocico corto y redondeado, ojos salientes con pupila horizontal. Su coloración era grisácea con manchas oscuras en cabeza y cuerpo, con variadas granulaciones en el dorso, todos atributos que permiten asignarlo a B. antartandica (Rabanal \& Núñez, 2008, p. 75). El ejemplar de B. antartandica se hallaba en la ribera de un río, aproximadamente a 180 metros al interior de isla desde el seno Skyring y a 16 metros sobre el nivel del mar. Al momento de su observación, estaba sobre la corteza de un árbol

1 Asociación Kauyeken (2018) "Parque Nacional Kawésqar. Refugio de vida". Video disponible en www.youtube.com/ watch?v=8x7Q0PAPduk

1 Estancia Anita Beatriz, Isla Riesco, Magallanes, Chile

2 Asociación Kauyeken, Isla Riesco \& Santiago, Chile

Ignacio Carrera Pinto 139, Punta Arenas, Chile

Outdoor TV, Santiago, Chile

Facultad de Ciencias, Universidad de Chile, Santiago, Chile. $\bigotimes$ jsimonet@uchile.cl 


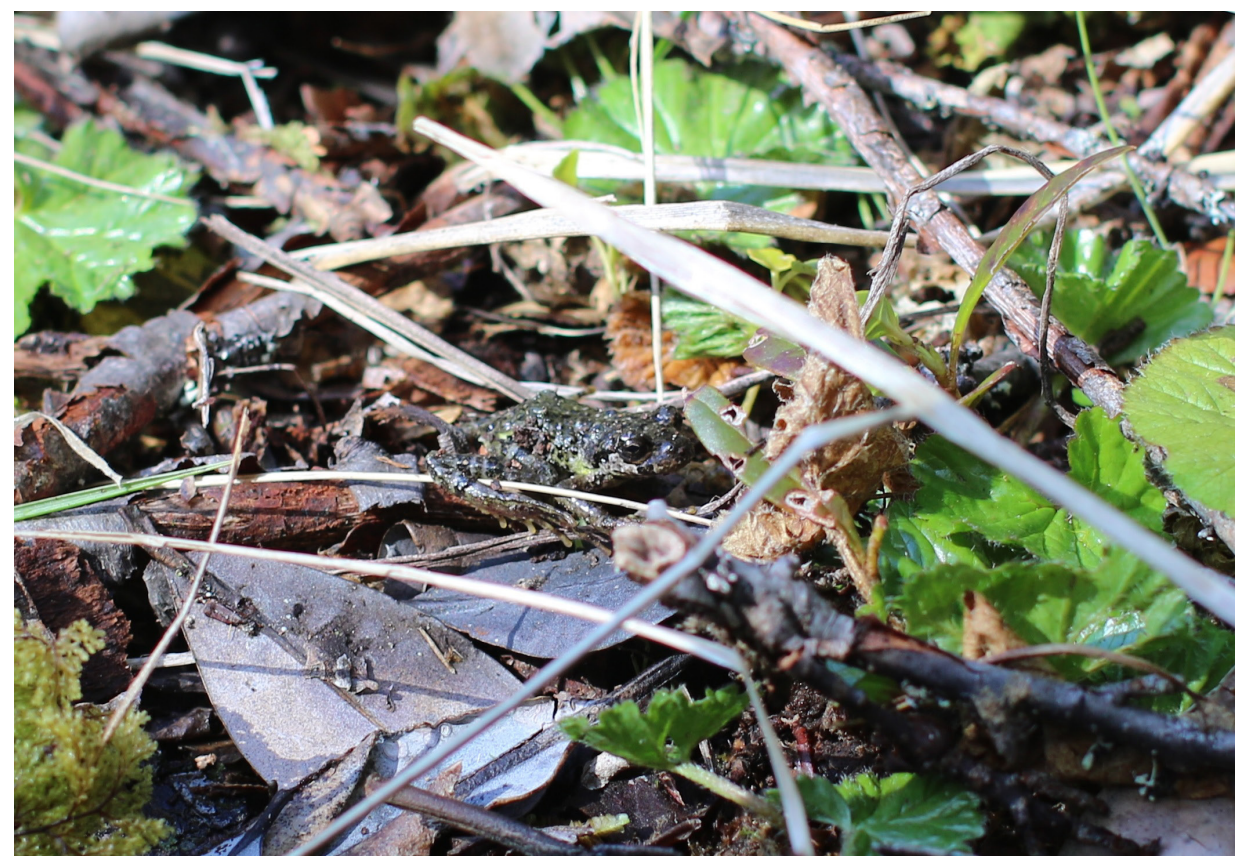

Fig. 1. Ejemplar de Batrachyla antartandica, Cabo León, Parque Nacional Kawésqar, Isla Riesco (enero de 2018).

caído, en un bosque siempreverde compuesto principalmente por coigüe de Magallanes (Nothofagus betuloides), canelo (Drimys winteri) y sauco del diablo (Raukaua laetevirens). El suelo estaba cubierto por abundante hojarasca y frutilla del diablo (Gunnera magellanica).

Los ejemplares de Isla Virtudes no concordarían plenamente con las características de B. antartandica (Atalah \& Sielfeld, 1976). El único ejemplar registrado en Isla Riesco no permite contribuir a resolver si las diferencias observadas en aquellos de Islas Virtudes se corresponden a variaciones intraespecíficas o si debería erigirse un nuevo taxón para estas poblaciones.

El hallazgo de B. antartandica corresponde a la segunda especie de anfibio registrada en Isla Riesco. Un adulto de Chaltenobrachus aff. grandisonae $\mathrm{y}$, presumiblemente, sus larvas han sido registrados en Estero Toro (53⒉' 'S; 72 34'O; Aguayo, 2007). Este hallazgo, posteriormente asignado erróneamente a Batrachyla sp, fue confirmado como $C$. aff. grandisonae (Frangópulos, 2007; Ortiz, 2015; detalles en Simonetti \& Stipicic, 2019). Estos dos recientes registros en Isla Riesco ponen nuevamente de manifiesto que se desconoce la riqueza de especies y los rangos de distribución de muchas especies ya conocidas en Magallanes (Asencio et al. 2009) y sugieren la necesidad de realizar inventarios de la biota de Isla Riesco (Simonetti \& Stipicic, 2019), incluyendo el nuevo Parque Nacional Kawésqar.

\section{AGRADECIMIENTOS}

Agradecemos a Andrés Charrier la confirmación de la identidad de B. antartandica. Las labores de campo fueron financiadas por J.M. Kaplan Fund.

\section{LITERATURA CITADA}

Aguayo, A. (Ed.) (2007). Informe Final "Diagnóstico de la Flora y Fauna de vertebrados y Aves marinas del AMCP Francisco Coloane". Punta Arenas, Chile: Ministerio de Bienes Nacionales.

Asencio, J., Kusch, A., Henríquez, J. M., \& Cárcamo, J. (2009). Registro de anfibios en el bosque Norpatagónico costero de Canal Messier, Chile. Anales del Instituto de la Patagonia, 37, 113-116.

Atalah, A., \& Sielfeld, W. (1976). Presencia 
de Batrachyla antartandica Barrio en Magallanes. Anales del Instituto de la Patagonia, 7, 168-170.

Barrio, A. (1967). Batrachyla antartandica n. sp. (Anura, Leptodactylidae) descripción y estudio comparativo con la especie genotípica, $B$. leptopus Bell. Physis, 27, 101-109.

Correa, C., Donoso, J. P., \& Ortiz, J. C. (2016). Estado de conocimiento y conservación de los anfibios de Chile: una síntesis de los últimos 10 años de investigación. Gayana, 80, 103-124.

Cuevas, C., \& Ugarte, Y. (2008). Geographic Distribution. Batrachyla antartandica. Herpetological Review, 39, 233.

Díaz-Páez, H., \& Young, S. (2003). Geographic Distribution. Batrachyla antartandica. Herpetological Review, 34, 379-380.

Formas, J. R. (1979). La Herpetofauna de los Bosques Temperados de Sudamérica. En W. E. Duellman (Ed.), The South American Herpetofauna. Museum of Natural History, University of Kansas. Monograph, 7, 341369.

Formas, J. R., \& Pugin, E. (1971). Reproducción y desarrollo de Batrachyla antartandica (Anura, Leptodactylidae). Boletín del Museo Nacional de Historia Natural, 32, 201-213.

Frangópulos, M. (2007). Diagnóstico de flora, fauna terrestre y aves marinas en el Área Marina Costera Protegida Francisco
Coloane. Punta Arenas, Chile: Informe Final, Fundación CEQUA.

Ortiz, J. C. (2015). Anfibios de las turberas del extremo austral de Chile. Cap. 8 pp. 229 - 240. En E. Domínguez \& D. Vega-Valdés (Eds.), Funciones y servicios ecosistémicos de las turberas en Magallanes. Punta Arenas, Chile: Instituto de Investigaciones Agropecuarias, Centro Regional de Investigación Kampenaike.

Penna, M. (1997). Selectivity of evoked vocal responses in the time domain by frogs of the genus Batrachyla. Journal of Herpetology, 31, 202-217.

Penna, M., Feng, A. S., \& Narins, P. M. (1997). Temporal selectivity of evoked vocal responses of Batrachyla antartandica (Amphibia: Leptodactylidae). Animal Behavior, 54, 833-848.

Rabanal, F. E., \& Núñez, J.J. (2008). Anfibios de los bosques templados de Chile. Valdivia, Universidad Austral de Chile.

Simonetti, J. A., \& Stipicic, G.J. (2019). Fauna terrestre de Isla Riesco, Magallanes: guía bibliográfica. Anales del Instituto de la Patagonia, 47, 7-18.

Úbeda, C., Veloso, A., Núñez, H., Díaz-Páez, H., Lavilla, E., \& Basso, N. (2004). Batrachyla antartandica. The IUCN Red List of Threatened Species 2004, e.T56330A11462162. Descargado 14 Noviembre 2019. 